\title{
Beyond Rigidity: Obtain Localizability with Noisy Ranging Measurement
}

\section{Xiaoping Wang*}

School of Computer Science,

National University of Defense Technology, Changsha, Hunan, PR China

Email: xiaopingwang@nudt.edu.cn

* Corresponding author

\section{Zheng Yang}

Department of Computer Science and Engineering,

Hong Kong University of Science and Technology, Clear Water Bay, Kowloon, Hong

Kong

Email: yangzh@cse.ust.hk

\section{Jun Luo and Changxiang Shen}

School of Computer Science,

National University of Defense Technology, Changsha, Hunan, PR China

Email: junluo@nudt.edu.cn

Email: cxshen@nudt.edu.cn

\begin{abstract}
Location awareness is crucial for wireless networks, attracting many research efforts in recent years. One of the fundamental problems of localization is to determine whether or not a network is uniquely localizable. To address this problem, existing approaches often adopt the rigidity theory, which assumes accurate inter-node distance measurements. Consequently, rigidity-based approaches are infeasible in case of ranging errors. To overcome such difficulty, we introduce the concept of strong localizability and propose an algorithm, called LAS, to identify and locate a kind of strongly localizable networks. Taking the geometric constraints into account, LAS is more realistic than existing rigidity-based approaches. We conduct extensive simulations and the results show that LAS can entirely localize a network of the average degree about 12 . In the same time, the average position error is about $3 \%$ of distance measurement range, when the ranging error is bounded by $10 \%$ to the real value of distance measurement.
\end{abstract}

Keywords: Flip ambiguity; Localization; Strongly localizable network; Wireless networks.

Reference to this paper should be made as follows: Wang, X., Yang, Z., Luo, J., and Shen, C. (2010) 'Beyond Rigidity: Obtain Localizability with Noisy Ranging Measurement', Int. J. Ad Hoc and Ubiquitous Computing: Special Issue on Wireless Network Algorithm and Theory, Vol. $\mathrm{x}$, No. $\mathrm{x}, \mathrm{pp}$. X-x.

Biographical notes: Xiaoping Wang received his BS and MS degree in the School of Computer Science from National University of Defense Technology, China, in 2003 and 2006, respectively. $\mathrm{He}$ is now a Ph.D. candidate in the School of Computer Science at National University of Defense Technology. His research interests include wireless sensor networks and operating system. He is a student member of the IEEE.

Zheng Yang received his B.S. degree in computer science, Tsinghua University, Beijing, China, in 2006. He is currently a Ph.D. candidate at the Department of Computer Science and Engineering, Hong Kong University of Science and Technology. His research interests include wireless ad-hoc/sensor networks, and pervasive computing. He is a student member of the IEEE and the ACM.

Jun Luo received his BS degree in the Computer School from Wuhan University, China, in 1984, and an MS degree in the School of Computer Science at National University of Defense Technology, China, in 1989. He is now a professor in the School of Computer Science at National University of Defense Technology. His research interests include operating system, parallel computing, security, and wireless sensor networks.

Changxiang Shen received his BS degree in the Department of Mathematics from Zhejiang University, China, in 1965. He is an academician of Chinese Engineering Academy. He is now 
an adjunct professor in the School of Computer Science at National University of Defense Technology. His research interests include operating system, parallel computing, security, and wireless sensor networks.

\section{Introduction}

Location awareness is crucial for wireless networks (Li and Liu, 2009), which provides essential contexts for data interpretation (Mo et al., 2009) and network operations (Li et al., 2008). Due to the constraints of hardware and cost, location information is not an initially available knowledge. Instead, only a few nodes, called anchors, know their locations in the network setup step. Then, other nodes compute their locations based on inter-node distance measurements. This procedure is called network localization. For localization, a fundamental problem is to determine the localizability of a network: whether or not the locations of the nonanchor nodes can be uniquely determined, given the distancemeasurement and anchor information.

Recently, an increasing number of researchers study the localizability issue and achieve many results on identifying localizable networks (Aspnes et al., 2006, Eren et al., 2004). However, those results are based on rigidity theory, which relies on two essential assumptions. First, rigidity theory requires accurate ranging. Second, it demands the node distribution to be generic, which means any group of nodes do not lie in a proper subspace (e.g., any three nodes do not lie on a line). Unfortunately, neither of the assumptions can be fully satisfied in practice. Hence, rigidity theory can only indicate localizability in theory.

Figure 1 (a) shows the ground truth of a network, where vertices denote the nodes and edges denote the associated nodes can measure the mutual distances. If all distance measurements contain no error, this network is localizable according to rigidity theory. However, error is inevitable in practice for all ranging techniques and hardware platforms. Suppose the ranging error is not more than $10 \%$ of the real distance values. If error is considered, the originally localizable graph in Figure 1 (a) has an alternative embedding that also satisfies all distance constrains shown in Figure 1 (b). In this case, the existence of two feasible embeddings destroys the localizability of the example network. Researchers usually use the stress to evaluate the fidelity of embeddings (Goldenberg et al., 2006), defined as the squared discrepancy between the induced inter-node distances and the measured distances, i.e. $\sum_{(i, j) \in E}\left(\left\|p_{i}-p_{j}\right\|-\hat{d}_{i j}\right)^{2}$, where $E$ is the edge set, $p_{i}$ is the realized position of vertex $i$, and $\hat{d}_{i j}$ is the measured distance between node $i$ and node $j$. In this example, the stress of Figure 1 (a) is 4.1. In contrast, the stress of Figure 1 (b) is 1.2 and will be chosen as the localization result, leading to huge errors in location estimation. To summarize, purely structural rigidity fails to guarantee the network localizability under noisy measurement.

To highlight the impact of errors on localizability, we define a network is weakly localizable if the network is localizable under accurate ranging information. Rigidity theory can be used to solve this type of localizability. In contrast, a network is strongly localizable if the network has a unique realization under noisy ranging measurement with bounded errors. In Section 2, we will give a formal definition of strongly localizable network, and show that a strongly localizable network has a unique realization in case of errors.
Figure 1 Two feasible realizations of a globally rigid graph with noisy ranging measurements

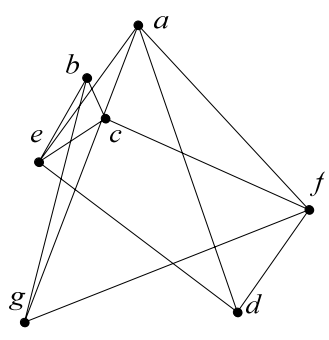

(a)

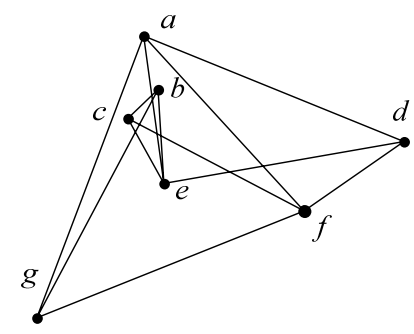

(b)
According to the theory of strong localizability, we propose a Localization Algorithm for Strongly localizable network, called LAS, to identify and locate a kind of strongly localizable networks: robust trilateration network. The insight of LAS is the equivalent conversion of the ranging errors. Compared with the existing robust localization approaches, LAS has the following characteristics. First, LAS introduces very low cost on the localization performance. Here, we define the performance of localization algorithms as how many nodes can be successfully located in a given network. In other words, a high-performance algorithm can work well in sparse networks. The experiment results show that LAS can achieve a full localization when the average degree is about 12. This result is of the same level with the traditional trilateration-based methods (Moore et al., 2004), which means LAS brings little performance degradation. Second, LAS is distributed and introduces low cost. The communicational and computational cost of LAS are both $O(n)$ in a network with bounded node degree, where $n$ is the number of nodes in the network. From the perspective of a single node, LAS only introduces $O(1)$ communicational and computational cost.

\section{Major contributions of this work are as follows.}

1. We propose the concept of strong localizability which accepts errors when studying localizability.

2. We propose two versions of localization algorithm (LAS and CLAS) to identify and locate robust trilateration networks, a special kind of strongly localizable networks. They both introduce low computational and communicational cost, thus are applicable in a resource-constrained network.

3. We conduct extensive simulations to evaluate our design. The results show that LAS and CLAS can achieve robustness with very low performance cost.

The rest of this paper is organized as follows. We formally define the strongly localizable network and robust trilateration network in Section 2. In Section 3, we describe the rationale of LAS. In Section 4, we describe the implementation of LAS and CLAS design. Experimental studies are given in Section 5. We review the related work in Section 6 and conclude this work in Section 7. 
Figure 2 Analysis of flip ambiguity

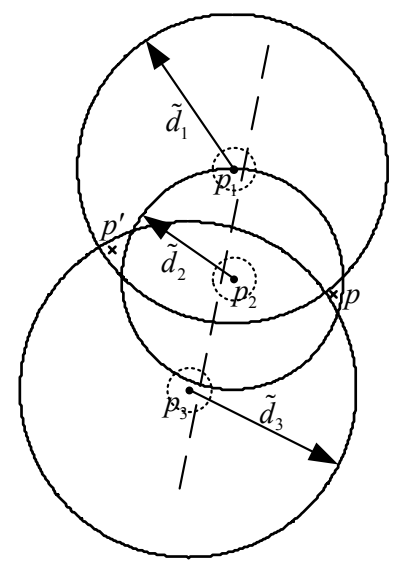

\section{Preliminary}

In this section, we present the network model and the formal definition of strongly localizable network. Moreover, we also discuss the concept of robust trilateration network.

\subsection{Strongly Localizable Network}

Given a network and corresponding distance measurements, we use a distance graph $G=(V, E)$ to present the measurements, in which the vertices in $V$ denote the nodes in the network and each edge $(i, j) \in E$ denotes node $i$ and node $j$ can measure the mutual distance. The corresponding measurement value and maximum error of each edge are presented by two functions: $d(i, j): E \rightarrow R$ and $\delta(i, j): E \rightarrow R$, respectively. We suppose a small portion of nodes, called anchors, are at known locations. Without loss of generality, $m$ anchors are labeled from 1 to $m$, together with $n-m$ ordinary nodes labeled from $m+1$ to $n$, where $n$ denotes the total number of nodes in the network. The ground truth position of each node is denoted by $p_{i}, 1 \leq i \leq n$. Hereinafter, we use a distance graph $G$ as well as the two functions $d$ and $\delta$, i.e. $(G, d, \delta)$, to model a given network.

A feasible realization of a network is a mapping $P: V \rightarrow R^{2}$ that guarantees ||$|P(i)-P(j) \|-d(i, j)| \leq \delta(i, j)$ for all $(i, j) \in E$, where $\|$.$\| denotes the Euclidean distance of two positions. We say a$ feasible realization is globally rigid, i.e. weakly localizable, if the distance-preserved mapping is unique in a plane (Aspnes et al., 2006). Then, we utilize this concept to define the strong localizability of a network.

Definition 1. A network is strongly localizable if all of its feasible realizations are globally rigid.

Strongly localizability is an extension of weak localizability. Weak localizability purely concerns the qualitative attribute of the network structure, i.e. stability. In contrast, strong localizability also emphasizes the quantitative attribute of the network structure. Clearly, weak localizability is a special case of strong localizability, where the bound of error $\delta(i, j)=0$ for all $(i, j) \in E$. The ground truth of a network must be one of the feasible realizations of the corresponding graph $(G, d, \delta)$. Hence, if a network is strongly localizable, the localization result will not suffer from the structural deformation.

\subsection{Robust Trilateration Network}

Location computation of weakly localizable networks is NP-hard
(Aspnes et al., 2006), thus location computation of strongly localizable network is also NP-hard, motivating the research on location-computable networks (Fang et al., 2009). Among them, the trilateration network is well studied. Measuring the distances to 3 reference nodes at known locations, a target node can uniquely determine its location through trilateration. A network is call trilateration network if it can be located by a series of trilaterations. To obtain the computability and strong localizability simultaneously, we propose the concept of robust trilateration network.

Definition 2. A robust trilaterative ordering for a graph $G$ is an ordering of the vertices $1,2, \ldots, n$, such that, (1) the first $m$ vertices are anchors, and (2) from every vertex $i>m$, there are at least 3 edges to vertices earlier in the sequence, and all the induced subgraphs of the first $i, m<i \leq n$, nodes are strongly localizable. A graph with a robust trilaterative ordering is called a robust trilateration graphs. And its corresponding network is a robust trilateration network.

In the two dimensional case, there are only two ambiguities preventing a rigid graph to be uniquely realized: flip ambiguity and flex ambiguity (Goldenberg et al., 2006). Fortunately, in trilateration graph, there is no chance for flex ambiguity, because redundantly rigidity is always satisfied in trilaterative extension. Hence, avoiding flip ambiguity is essential for achieving a robust trilateration network. Flip ambiguity is defined as that a target node has two possible positions corresponding to a "reflection" across a set of mirror nodes (Goldenberg et al., 2006). Figure 2 shows an example of flip ambiguity in trilateration. When the reference nodes $\left(p_{1}, p_{2}\right.$, and $\left.p_{3}\right)$ are approximately collinear, it is ambiguous to determine whether position $p$ or $p^{\prime}$ (denoted by $\mathrm{x}-$ marks) is the correct location estimation of the target node.

To obtain a robust trilateration network, we first generate a subnetwork of all anchors, and then extend the sub-network by iteratively finding a node that fulfills the following conditions: (1) the node has at least three distance measurements to nodes already in the sub-network; and (2) the node can calculate its location without flip ambiguity.

\section{Analysis of the Flip Ambiguity with Noisy Ranging Measurement}

In this section, we first discuss the rationale of the design of LAS, especially the idea of equivalent translation of the ranging errors. Then, we analyze the translated error and solve the problems along with it.

\subsection{Equivalent Translation of Errors}

We first show the equivalent translation of the measurement errors. Suppose we localize a target node by $k(k \geq 3)$ distance measurements to the nodes at known locations, called reference nodes. Let a set of tuples $M=\left\{<p_{i}, \tilde{d}_{i}>\right\}, i=1,2, \ldots, k$, to denote the reference nodes, where $p_{i}$ denotes the position of reference node $i$, and $\tilde{d}_{i}$ denotes the measured distance between the target node and the reference node $i$. Each $\tilde{d}_{i}$ contains two parts: the real distance between the node pair of $d_{i}$ and the error of the measurement $\varepsilon_{i}$, i.e. $\tilde{d}_{i}=d_{i}+\varepsilon_{i}$. Suppose the exact position of the target node is given. We can translate the reference node $\varepsilon_{i}$ distance along the direction of the distance measurement and we get the translated position $\tilde{p}_{i}$. After this step, we obtain a group of new measurement tuples $M^{\prime}=\left\{<\tilde{p}_{i}, \tilde{d}_{i}>\right\}, i=1,2, \ldots, k$. The only difference between $M$ and $M^{\prime}$ is that there are no measurement 
errors in $M^{\prime}$. As a result, if all $k$ translated positions $\tilde{p}_{i}$ in $M^{\prime}$ are not likely to be collinear, there is no chance of flip ambiguity.

In practice, neither the real position of the target node nor the measurement errors are available. Moreover, the positions of reference nodes may also contain errors. Hence, we cannot obtain the exact form of $M^{\prime}$. Instead, we can estimate it by the distance measurement error and position estimation error. For a reference node $i$ in $M$, suppose the maximum absolute value of the measurement error is $\delta_{d, i}$, and the maximum absolute value of position estimation error is $\delta_{p, i}$. Note that both of them may be a function of distances and other environmental parameters (Patwari et al., 2005). The translated position $\tilde{p}_{i}$ must lie in the equivalent disk, which is defined as a disk centered at $p_{i}$ with a radius $r_{i}=\delta_{d, i}$ $+\delta_{p, i}$. Then, we rewrite the tuples $<\tilde{p}_{i}, \tilde{d}_{i}>$ in $M^{\prime}$ to this form $<p_{i}, r_{i}, \tilde{d}_{i}>$. Let $\tilde{M}$ denote the set of the equivalent measurement, $\tilde{M}=\left\{<p_{i}, r_{i}, \tilde{d}_{i}>\right\}, i=1,2, \ldots, k$, which contains the possible positions of translated reference nodes and exact distance information.

Let $p$ and $p^{\prime}$ denote the two possible positions due to a flip in M, then the perpendicular bisector of line segment $p p^{\prime}$ must pass through all $\tilde{p}_{i}$ in $M^{\prime}$. Hence, the perpendicular bisector must intersect with all equivalent disks in $\tilde{M}$. As shown in Figure 2, we localize a target node by three measurements to the nodes indexed by 1,2 , and 3 , respectively. If the two candidate position denoted by $\mathrm{x}$-mark are ambiguous by trilateration, then the perpendicular bisector of them (the dashed line in Figure 2) intersects with all the equivalent disks (the dashed circles in Figure 2). Hence, we obtain a property of flip ambiguity: when the trilateration suffers from flip ambiguity, the perpendicular bisector of the candidate positions must intersect with all equivalent disks of reference nodes.

This property provides an inspiration to avoid flips: if there does not exist any line that can intersect with all equivalent disks of reference nodes, then flip will not take place. For a given reference node set, we investigate the following existence of intersecting line (EIL) problem to estimate the risk of flip ambiguity:

Given: A set of disks $S=\left\{<p_{i}, r_{i}>\right\}, i=1,2, \ldots, k$, in a plane.

Objective: To determine whether there exists a line intersecting with all disks in $S$.

\subsection{EIL problem of equal radii}

The EIL problem of equal radii focuses on a special case in which all the given disks are of the same size. Solving this issue can answer two questions in localization: (1) when we indistinctively estimate the errors of the reference nodes, can the target node suffer from flip ambiguity? (2) when there is no clear bound of the errors, how much error can the reference node set tolerate for avoiding flip ambiguity?

Figure 3 Width of a set and the medial line

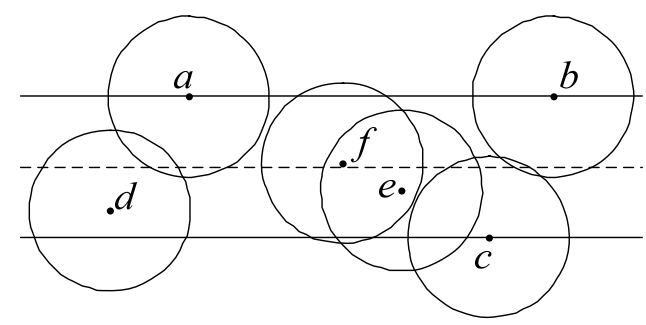

The following theorem shows the equivalent geometric property for solving the EIL problem with equal radii:

Theorem 1. Given a set of disks $S=\left\{<p_{i}, r_{i}>\right\}$, if all the disks in $S$ are of the same size, i.e. $r_{i}=r, i=1,2, \ldots, k$, the EIL problem is equivalent to determine whether the width of the centers $P=\left\{p_{i}\right\}$, denoted as $W(P)$, is larger than $2 r$. The width of $P$ is defined as the minimum distance between parallel lines of support of $P$.

Proof. Define the medial line of $P$ as the line whose distances are at most $W(P) / 2$ with the points in $P$. Take Figure 3 as an example, the distance of the solid lines shows the width of the point set, and the dashed line shows the medial line of the point set. If $W(P) \leq 2 r$, then the medial line will intersect all disks in $S$. Hence, there exists a line intersecting all disks. If there exists a line intersecting all disks in $S$, then the maximum distance between the line and the points in $P$ is $r$. Hence, the width of $P$ is less than or equal to $2 r$, i.e. $W(P) \leq 2 r$. In a word, the answer of EIL problem of equal radii is equivalent to the condition $r>W(P) / 2$.

We define the value $W(P) / 2$ as the error tolerance of a reference node set. The error tolerance is computed by the width of the reference node set $P$. There are several high efficient algorithms to compute the width of a point set, such as Rotating Caliper Algorithm (Houle and Toussaint, 1988). The algorithm mainly has three steps. First, we compute the convex hull of the point set. Clearly, the points determining the width of the set are definitely on the convex hull. Second, we find an arbitrary antipodal vertexedge pair. We call an edge and a vertex of the convex hull an antipodal vertex-edge pair if parallel lines of support of $P$ contain the edge and vertex. In Figure 3, vertex $c$ and edge $a b$ is an antipodal vertex-edge pair of the convex hull $a b c d a$. Third, we "rotate" the initial antipodal vertex-edge pair to enumerate all the antipodal vertex-edge pairs of the convex hull, and report the half of the minimum distance of the antipodal vertex-edge pairs as the error tolerance, as shown in Algorithm 1.

Suppose there are $k$ points in set $P$. Computing the convex hull of $P$ in step 1 leads a complexity of $O(k \log k)$ time. Since there are at most $k$ vertices or edges in $\mathrm{CH}$, finding the initial antipodal vertex can be done in $O(\log k)$ time using binary search. Finally, the rotating calipers method can generate all the antipodal vertexedge pairs in $O(k)$ time. Hence, the overall complexity of Algorithm 1 is $O(k \log k)$.

Algorithm 1 ErrorTolerance

Input: the positions of reference nodes $P$

Output: the maximum error which the reference node set can tolerate for flip ambiguity

1: Compute the convex hull of $P$

$\mathrm{CH}=$ ConvexHull $(P)$

2: Find initial antipodal vertex-edge pair

$e=$ an arbitrary edge in $\mathrm{CH}$

$v=\operatorname{BinarySearch}(e, \mathrm{CH})$

3: Use the rotating calipers to generate all antipodal vertex-edge pairs

while existing edges in $\mathrm{CH}$ that have not been rotated do $e=$ the clockwise neighboring edge of $e$ in $\mathrm{CH}$

Rotate $v$ clockwise until $(v, e)$ form a antipodal vertex-edge pair

Width $=$ the minimum distance of all the known $(v, e)$ pair

4: return $w i d t h / 2$ 
Figure 4 The boundary of intersecting line set

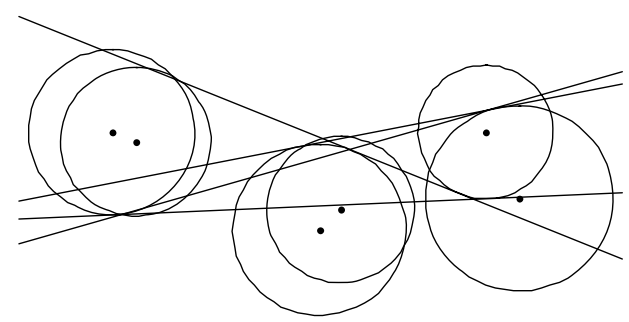

\subsection{EIL problem of unequal radii}

It is hard to solve the EIL problem of unequal radii by the macrostructure of the disks. Hence, we adopt boundary check to solve the EIL problem of unequal radii, as shown by Theorem 2 .

Theorem 2. Given a set of disks $S=\left\{\left\langle p_{i}, r_{i}>\right\}, i=1,2, \ldots, k\right.$, the EIL problem is equivalent to determine that whether there exists a common tangent of two disks in $S$ that intersects with all disks in $S$.

Proof. Let $I L(S)$ denote the intersecting line set of the disk set $S$. The EIL problem is to determine whether or not $I L(S)=\varnothing$. Clearly, if $I L(S)=\varnothing$, there is no common tangent of two disks in $S$ that intersects with all disks in $S$. Then, to analyze the case of $I L(S) \neq \varnothing$, we preprocess the disk set $S$. When a disk lies completely in another disk, any line intersecting with the inner disk will definitely intersect with the outer one. In this case, we can safely delete the outer disk from $S$. We use $S^{\prime}$ to denote the preprocessing result of $S$. Then, any pair of disks in $S^{\prime}$ have common tangents. In the following, we want to prove that there must exist a common tangent that intersects with all disks if $I L\left(S^{\prime}\right) \neq \varnothing$. The boundary of $I L\left(S^{\prime}\right)$ is a line that is about to violate the condition of intersecting with all disks in $S^{\prime}$. Hence, a boundary line must be tangential to one disk. If the line is not tangential to any other disk, we rotate the line round the centre of the disk till it reaches the border of another disk. In this case, the line becomes a common tangent of two disks in $S^{\prime}$ and intersects with all disks.

\section{Algorithm 2 EIL}

Input: the set of disks $S=\left\{<p_{i}, r_{i}>\right\} i=1,2, \ldots, k$

Output: a Boolean value that indicates whether there exists a line intersecting with all disks in $S$.

1: Delete the outer disk of overlapped disks $S^{\prime}=\operatorname{Preprocess}(S)$

2: The trivial case

if $\left|S^{\prime}\right| \leq 2$ then

return true

3: The extreme cases

$P^{\prime}=$ the set of the centers of disks in $S^{\prime}$

et $=\operatorname{ErrorTolerance}\left(P^{\prime}\right)$

if $e t \leq \min \left(\left\{r_{i}\right\}\right)$ then

return true

elseif $e t>\max \left(\left\{r_{i}\right\}\right)$ then

return false

4: Boundary checking

for each disk pair $d_{1}, d_{2} \in S^{\prime} d_{1} \neq d_{2}$ do

$C T=$ CommonTangent $\left(d_{1}, d_{2}\right)$

for each line $l \in C T$ do

if $l$ intersects with all disks in $S^{\prime}$ then return true

5: If it does not return in boundary checking step, then no intersecting line exists

return false
We show all boundary lines of a disk set in Figure 4. Based on Theorem 2, we propose an algorithm to solve the EIL problem with unequal radii, as shown in Algorithm 2.

Suppose there are $k$ disks in set $S$. Step 1 needs to check all disk pairs and leads a complexity of $O\left(k^{2}\right)$ time. The size of $S^{\prime}$ is at most $k$. Step 3 calls the ErrorTolerance procedure in Algorithm 1 and leads a complexity of $O(k \log k)$ time. In fact, this step is optional and helps to make a direct decision in two extreme cases. The outer loop of Step 4 needs to repeat $O\left(k^{2}\right)$ times. The maximum number of common tangents of two disks is four, i.e. $|C T| \leq 4$ in Step 4. Thus the inner loop of Step 4 repeats $O(1)$ times. Moreover, checking intersection of all disks costs $O(k)$ time. Hence, the boundary checking in Step 4 can be done in $O\left(k^{3}\right)$ time, and the overall complexity of Algorithm 2 is $O\left(k^{3}\right)$.

\section{Implementation of LAS Design}

The design of EIL test is a robust complement of traditional localization scheme. Hence, it can be directly applied into the traditional trilateration or the cluster-based localization algorithms. We call these two implementations LAS and CLAS, respectively.

LAS inherits the merits of trilateration (Goldenberg et al., 2006, Eren et al., 2004): easy to implementation and fully distributed. The distributed trilateration is implemented as follows. First, nodes measure and record the distances with direct neighbors through the ranging devices. Second, anchor nodes broadcast their locations to their direct neighbors. Third, each node overhears the location broadcast of its neighbors. If the node can collect at least three location broadcasts of its neighbors, it computes the location of itself by trilateration. Then, it broadcasts its location to its direct neighbors. Finally, by waiting sufficient long time, if a node still cannot collect three location broadcasts from its neighbors, it marks itself as an unlocalized node. LAS only need to enhance the third step to obtain robustness. In the third step, LAS requires the reference node set to pass the EIL test, before it performs a trilateration. Hence, LAS introduces very low additional cost when compared with traditional trilateration. If the number of neighbors in the network is bounded by a constant, the communicational cost and the computational cost are both $O(n)$, where $n$ is the number of nodes in the network.

CLAS adopts the clusters to relax the requirements of anchor distribution. CLAS first generates a local coordinate system of a cluster. Then, nodes join to the cluster by trilateration, localizing itself in the local coordinate system. Finally, LAS localizes the clusters having at least three anchors to the physical coordinate system by coordinate system registration (Horn et al., 1988). Clusters make the CLAS less depend on the anchor distribution, thus may increase the performance of localization in sparse networks. Nevertheless, the way generating and converting coordinate system may introduce new sources of errors, thus it may potentially increase the error of the result. To obtain robustness, CLAS also requires nodes pass the EIL test, when joining to the local clusters. Moreover, when CLAS localize the clusters, it demands the anchors to pass the EIL test to avoid global flip ambiguity. The detail of CLAS is shown in Algorithm 3.

\section{Experiment}

In this section, we conduct extensive simulations to evaluate the proposed algorithms. 


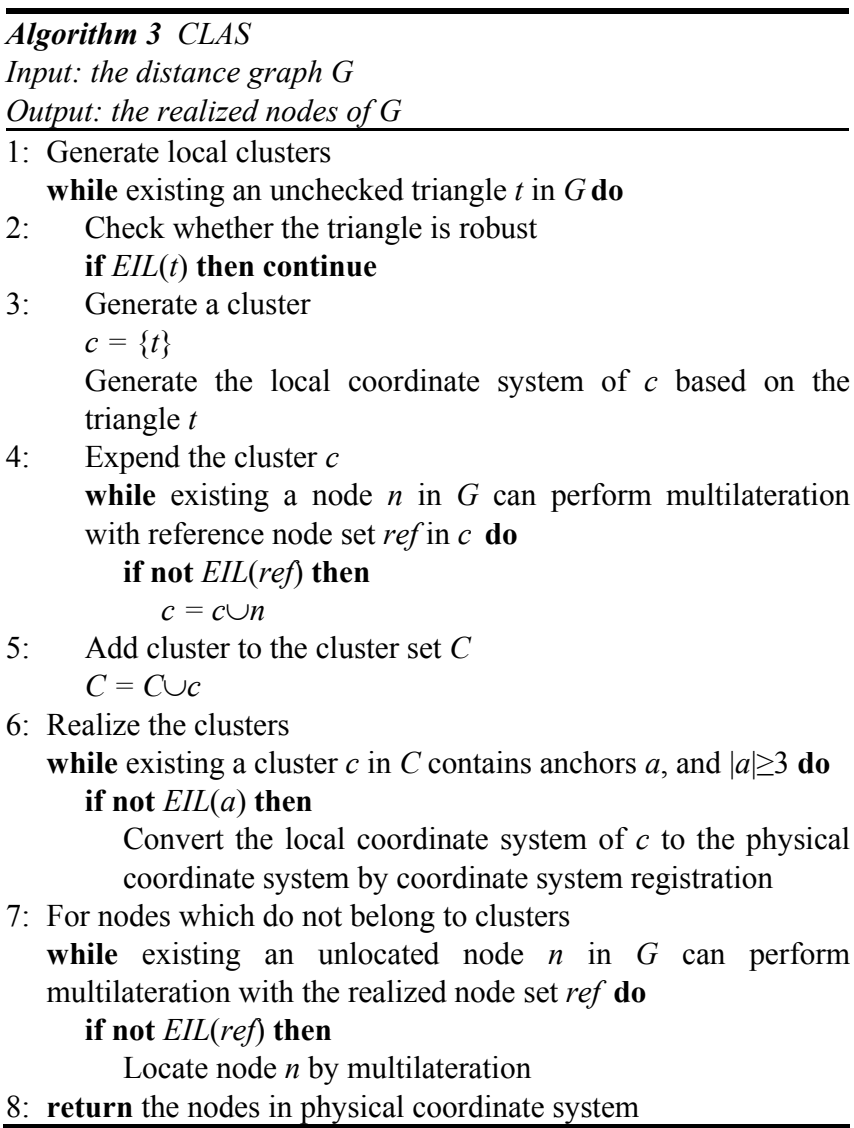

\subsection{Experiment Setup}

We generate uniformly random distributed network instances of 200 nodes in a square region. We randomly select a certain proportion of the deployed nodes as anchors. We use the parameter of distance measurement range to control the mean degree of the network. The distance information between neighboring nodes is corrupted by additive white Gaussian noise (Liu et al., 2006) $N\left(0, \sigma^{2}\right)$ and we bounded the error by $3 \sigma$. We control the following parameters to compare the efficiency of LAS and CLAS:

1. the mean degree of the network instances

2. the proportion of anchors in the network

3. the standard deviation of the distance-measurement noise

We evaluate the efficiency of LAS and CLAS in three aspects. First, the number of successfully localized nodes indicates the performance of each algorithm. Second, the standardized position estimation error (SPEE) shows the error of the result. SPEE is defined as the percentage value between the mean position estimation error and the maximum distance-measurement range:

$$
S P E E=\frac{1}{n R_{\max }} \sum_{i=1}^{n}\left\|p_{i}-\hat{p}_{i}\right\| \times 100 \%
$$

where $n$ is the total number of successfully localized nodes, $R_{\max }$ is the maximum distance measurement range, $p_{i}$ is the real position of node $i$, and $\hat{p}_{i}$ is the estimated position of node $i$. If $n=0$, we define SPEE $=0$. Third, the standardized distance estimation error (SDEE) indicates the fidelity between the estimated distance and the measured distance. SDEE is defined as the percentage value between the distance estimation error and the maximum distancemeasurement range:

$$
S D E E=\frac{1}{m R_{\max }} \sum_{i, j \in N, \tilde{d}_{i j} \in D}\left|\left\|\hat{p}_{i}-\hat{p}_{j}\right\|-\tilde{d}_{i j}\right|,
$$

where $N$ is the set of located nodes, $R_{\max }$ is the maximum distance measurement range, $D$ is the set of distance measurement, $\hat{p}_{i}$ is the estimated position of node $i$, and $m$ is the total number of such measurement pairs. If $m=0$, we define $\mathrm{SDEE}=0$.

We implement CLAS and LAS algorithm based on the error tolerance. We set the error tolerance threshold as the maximum possible error, i.e. $3 \sigma$. We compare the CLAS and LAS algorithms to the state-of-the-art design, robust quadrilaterals (RQ) (Moore et al., 2004). RQ shares the same design goal with us, which aims to acquire the structural stability with noisy ranging measurements. RQ acquires robustness by setting a threshold on the geometric property of the local four-node clusters. Stitching these robust local clusters, the resultant global cluster will not face structural deformation either. We implement full RQ algorithm with cluster optimization in the cluster generation step, and the clusters are merged by the coordinate system registration (Horn et al., 1988). In the following sections, we will investigate the performance of LAS and CLAS, as well as the impact of cluster-based method on the accuracy of localization.

\subsection{Overview of the Algorithms}

We demonstrate the execution of the RQ, LAS, and CLAS algorithm in a randomly generated network instance with 200 nodes, and the average degree is about 20 . We randomly select $10 \%$ nodes as anchors, and set the errors as much as $10 \%$ of the distance measurement value. Figure 5 shows the result of each algorithm, in which solid squares denote anchors, soft dots present the ground truth positions of the nodes, and the soft squares indicate the estimated positions of the nodes.

Figure 5 (a) shows the result of RQ algorithm. It locates 103 out of the total 180 non-anchor nodes. The SPEE is about 4.7 and the SDEE is about 2.6. Figure 5 (b) shows the result of LAS algorithm. It locates all the 180 non-anchor nodes. The SPEE is about 2.3 and the SDEE is about 1.3. Figure 5 (c) shows the result of CLAS algorithm. It locates all the 180 non-anchor nodes. The SPEE is about 2.7 and the SDEE is about 1.4.

The RQ algorithm locates fewer nodes than the other two algorithms, because it requires generating uniquely distributed overlapped local clusters. However, to guarantee robustness, RQ drops a large proportion of the generated local clusters. Hence, it requires high average degree to compensate the shortage of the local clusters.

The LAS algorithm gets the lowest SDEE and SPEE, because it does not base on clusters. Clusters may introduce errors both in generation step and coordinate system conversion step. In generation step, the local coordinate system is generated by the inter-node distances. When the inter-node distances contain errors, the corresponding local coordinate will be nonorthogonal and this can further influence all nodes in the cluster. In the coordinate system conversion step, the conversion is purely based on the position of anchors. This procedure will introduce additional errors for the nodes that are far from the anchor nodes, and the error of the conversion will be linearly amplified by the distance to anchors. 
Figure 5 Overview of the algorithms

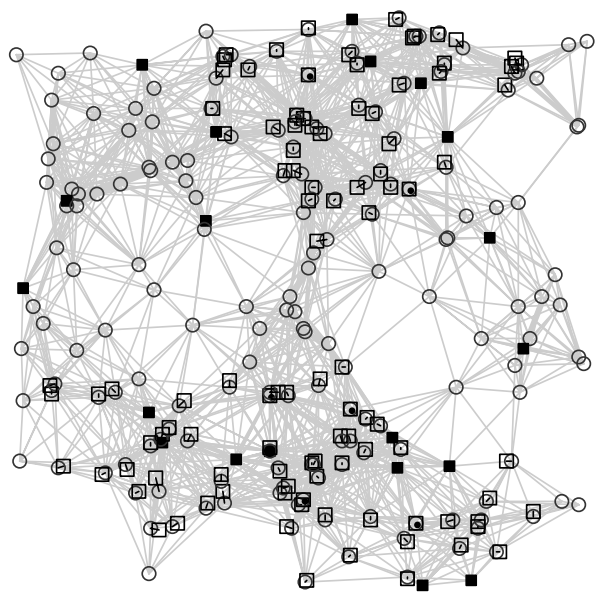

(a)

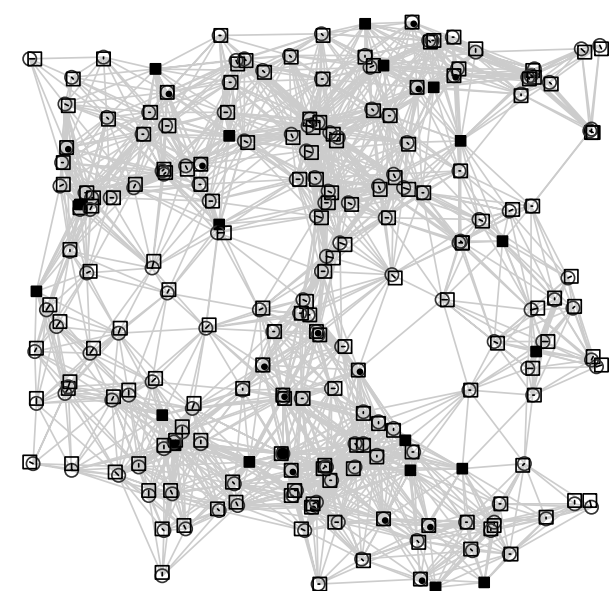

(b)

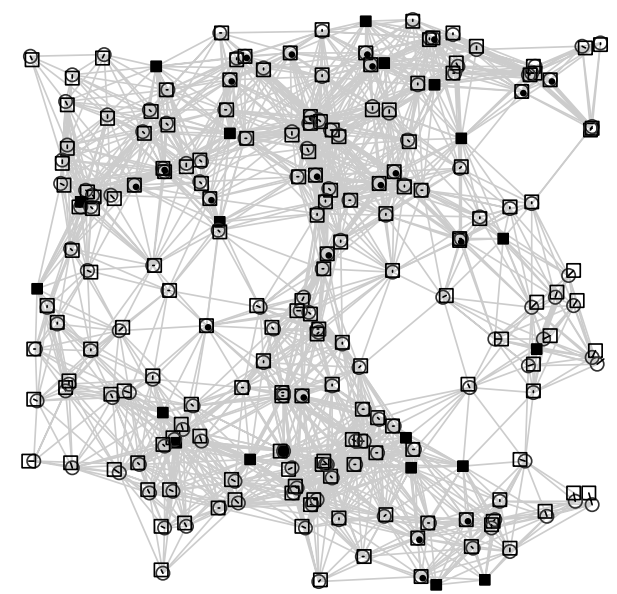

(c)

\subsection{The Impact of Average Degree}

In this section, we investigate the impact of average degree. We fix the anchor proportion to $10 \%$ and set the errors as much as $10 \%$ of distance measurement. We use an empirical formula to control the average degree of the network instances into approximately linear distribution. We set the step length to be about 0.5 and report the result in Figure 6.

Figure 6 (a) plots the proportion of localized nodes against average degree. When the average degree increases, all algorithms
Figure 6 The impact of average degree

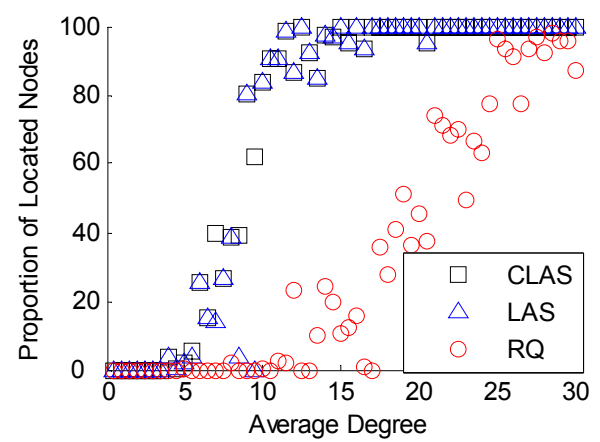

(a)

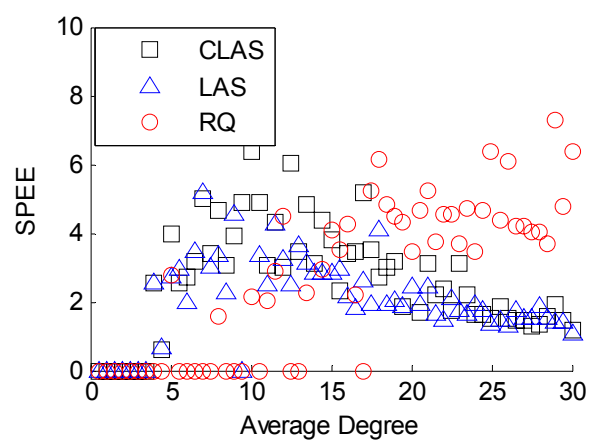

(b)

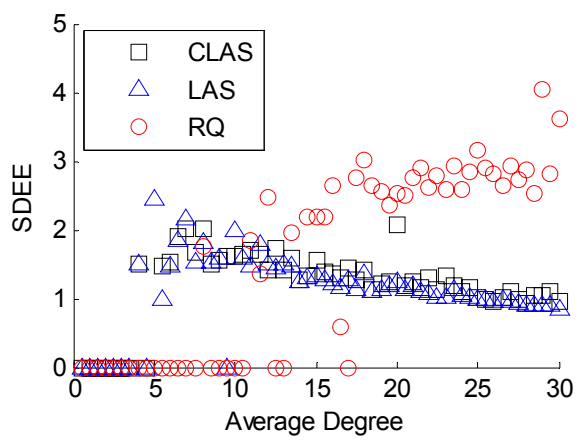

(c)

perform better. The LAS and CLAS algorithm can achieve 100\% localization when the average degree is greater than 12 . The CLAS algorithm outperforms the LAS algorithm when the average degree varies from 5 to 10 . In contrast, the RQ algorithm requires the average degree to be over 25 for full localization. RQ requires generating uniquely overlapped local clusters, thus RQ requires higher network density than the other two algorithms.

Figure 6 (b) and (c) plot the SPEE and SDEE against average degree, respectively. The SPEE and SDEE of the LAS and CLAS algorithm decrease when the average degree enlarges. Nevertheless, the SPEE and SDEE of RQ are quiet stable over all the tested range. This shows that the RQ algorithm cannot benefit from the increase of network density. No matter how many neighbors are available, RQ always generates four-node clusters and uses the six inter-node distances to form local coordinates. In contrast, LAS and CLAS can perform a more accurate estimation through all available ranging measurements, which provides better estimation than RQ does. 
Figure 7 The impact of anchor proportion

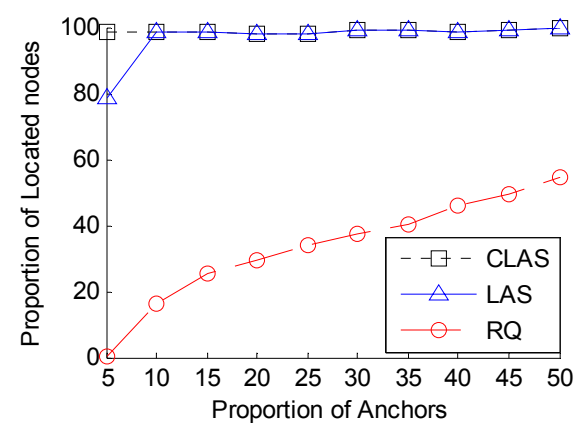

(a)

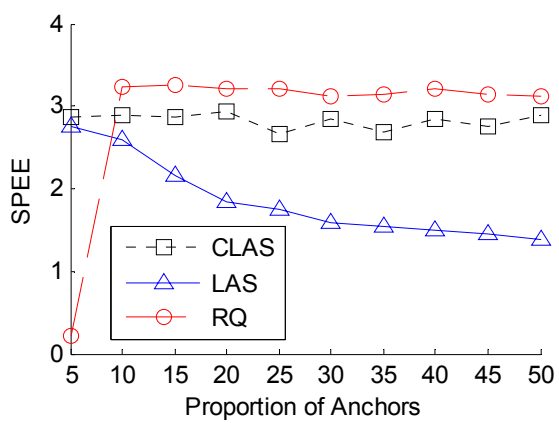

(b)

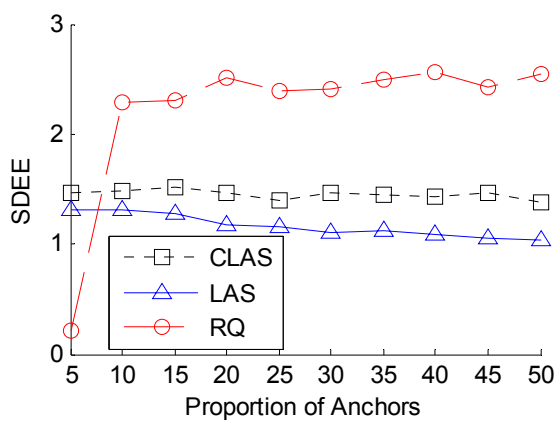

(c)

\subsection{The Impact of Anchor Proportion}

In this section, we investigate the impact of anchor density. We fix the average degree about 15 and set the errors as much as $10 \%$ of distance measurement. Figure 7 reports the mean value of 50 network instances in each anchor density configuration.

Figure 7 (a) plots the proportion of located nodes against anchor density. CLAS outperforms LAS when anchors are not adequate. For a high anchor density, they both can locate almost all nodes in the network and keep this level when anchor density increases. RQ performs better when anchor density enlarges, because more anchors can help to localize the local clusters.

Figure 7 (b) plots the SPEE against anchor density. The SPEE of LAS algorithm decreases when more anchors exist. However, the CLAS and RQ algorithm do not benefit from anchor density increase. The cluster-based method has the inherent limitations on the accuracy of the coordinate system generation and localization procedure as discussed before, and these errors cannot be reduced by anchors.
Figure 8 The impact of error magnitude

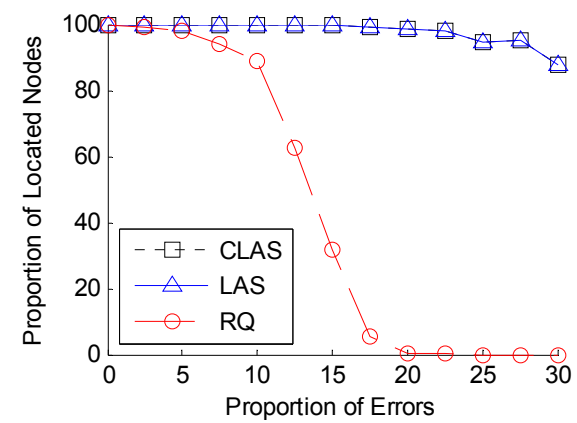

(a)

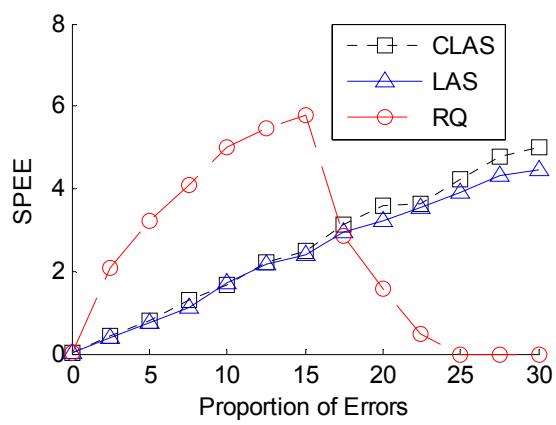

(b)

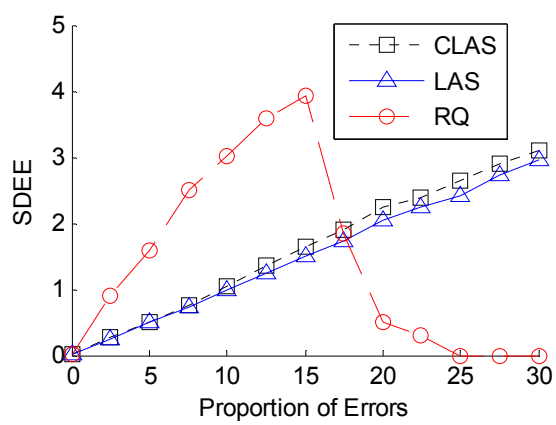

(c)

Figure 7 (c) plots the SDEE against anchor density. All the algorithms have little change when anchor density varies, because SDEE indicates the mean distance estimation error. This error is dominated by the error of distance measurement, so that the anchor density does not influence it. We observe that both SPEE and SDEE of RQ are quiet low at the point $5 \%$ anchors. We define the SPEE and SDEE is zero when no nodes are successfully localized, thus the values of SPEE and SDEE will be low when RQ fails locating some of network instances.

\subsection{The Impact of Error Magnitude}

In this section, we investigate the impact of error magnitude. We fix the anchor proportion to $10 \%$ and set the average degree about 25 . Figure 8 reports the mean value of 50 network instances in each configuration of the maximum error proportion.

Figure 8 (a) plots the proportion of located nodes against the standard deviation of the ranging error. All the algorithms perform worse when the measurement errors enlarge, because higher error will make more trilateration non-robust for flip ambiguities, thus to prevent some nodes to be located. High error affects RQ more, 
because RQ relies on overlapped local clusters to process localization. Higher error will affect the generation of local clusters in cluster generation step, and then influence the whole following procedure.

Figure 8 (b) and (c) plot the SPEE and SDEE against error magnitude, respectively. For the LAS and CLAS, the SPEE and SDEE are approximately linear with the error magnitude. The SPEE and SDEE of RQ are linear with the error, when the error is less than $15 \%$ of the distance measurement. Then, they start to decrease, because the SPEE and SDEE will be zero when no nodes are located in some of the network instances.

\section{Related Work}

Localization in wireless networks has attracted significant research interest in recent literatures (Liu et al., 2010). In this section, we briefly review some typical works in this area, including the localization theory and the error control algorithms.

\subsection{Theory of Network Localization}

Recently, a theory of network localization is proposed to investigate network localization problem (Aspnes et al., 2006). First, Aspnes et al. show the NP-hardness of the network localization problem and propose to investigate the localization problem in sparse networks (Aspnes et al., 2004). Then, Eren et al. adopt rigidity theory to investigate the network localizability problem (Eren et al., 2004, Goldenberg et al., 2005). Afterwards, Fang et al. propose to bridge the gap between the localizable network and location computation by the concept of sequential localization (Fang et al., 2009). Finally, based on localization theory, a series of algorithms are proposed to achieve efficiency on localization (Goldenberg et al., 2006, Wang et al., 2008). In addition, some algorithms also utilize rigidity theory (Lederer et al., 2008, Wang et al., 2009, Priyantha et al., 2005) to achieve other design goals. Nevertheless, rigidity theory is not so applicable to model practical localization problem; because the fundamental assumption of rigidity theory is that the distances between the nodes are accurate. The ranging measurement, however, is always contains errors in practice (Moore et al., 2004). To the best our knowledge, there are no works that adopt the practical model for the localizability problem. In a word, our design is an extension on both theory and algorithm of network localization.

\subsection{Error Control in Localization}

There is a large category of localization algorithms aiming to diminish the errors in the result (Ni et al., 2004, Shang and Ruml, 2004, Lim and Hou, 2005, Niculescu and Nath, 2004, Savvides et al., 2003, Savvides et al., 2005, Whitehouse et al., 2005, Li and Liu, 2010, Liu et al., 2008, Liu et al., 2007), when the ranging information is not accurate. Moore et al. propose the concept of robust quadrilaterals to avoid flip ambiguity with noisy ranging measurement (Moore et al., 2004). They choose robust geometric structures in the network as the basic localization units to guarantee the structural uniqueness of located nodes. Besides, Kannon et al. complement this work by general geometric analysis (Kannan et al., 2007, Kannan et al., 2008). To the best of our knowledge, this design is the most recent work to achieve structural uniqueness with noisy inter-node distances. However, their approach is too aggressive for flip ambiguity prediction, so that it will greatly decrease the localization performance. Moreover, simulation result show that based on the small-scale clusters will suffer error accumulation in the result. In contrast, LAS can achieve structural uniqueness as well as high localization performance.

Liu et al. (Liu et al., 2006) utilize a probabilistic model to estimate and control errors of trilateration. They evaluate the possible errors in each trilateration and select robust reference nodes to minimize the estimated error of the result (Yang and Liu, 2010). Their design mainly focuses on the overall estimation error of the result. Nevertheless, the estimation of error will not exclude the structural deformation of the result, thus their approach provide no guarantee on the localizability of the result. In contrary, LAS can guarantee the structural uniqueness of the result and the error of the result is proportional to the ranging errors. Fortunately, we can adopt error control in LAS to benefit from both of the advantages, i.e. the structural stability and better error control.

Basu et al. propose an iterative method to locate nodes with noisy distance and angle information (Basu et al., 2006). They use linear programming to address the relaxed form of the raised problem. Their design can also guarantee to avoid structural deformation and also provide the error estimation in the result set. However, this design relies on the knowledge of both distance and angle measurement of the neighboring nodes, which are not easy to acquire in practice.

Some researchers utilize the Cramer-Rao lower bound (CRLB) to characterize the error of network localization (Niculescu and Nath, 2004, Savvides et al., 2003, Patwari et al., 2005, Savvides et al., 2005). CRLB provides a lower bound on the variance achievable of an unbiased location estimator (Patwari et al., 2005). However, all probabilistic methods, including CRLB, cannot guarantee structural uniqueness of localization as discussed in Section 2. Further, CRLB requires the estimator to be unbiased, which is not so realistic for the off-the-shelf location estimators.

\section{Conclusions \& Future Works}

In this paper, we introduce the concept of strong localizability to achieve the structural stability for networks with noisy ranging measurements. We also propose an algorithm, called LAS, to identify and locate a kind of strongly localizable network: robust trilateration network. Compared with existing approaches, LAS introduces lower cost on performance and controls error better. Extensive experiments are conducted to evaluate the efficiency of the proposed algorithms.

The future work leads into two directions. First, we will design low complexity algorithms for EIL test of unequal radii. Second, we will investigate how to obtain strong localizability in a general network. Currently we are implementing this design in our ongoing projects.

\section{Acknowledgements}

This work is supported in part by NSF China 60970118 and 60903224

\section{References}

ASPNES, J., EREN, T., GOLDENBERG, D. K., MORSE, A. S., WHITELEY, W., YANG RICHARD YANG, ANDERSON, B. D. O. \& BELHUMEUR, P. N. (2006) A Theory of Network Localization. IEEE Transactions on Mobile Computing, 5(12), 1-15. 
ASPNES, J., GOLDENBERG, D. \& YANG, Y. R. (2004) On the Computational Complexity of Sensor Network Localization. Algorithmic Aspects of Wireless Sensor Networks: First International Workshop (ALGOSENSORS).

BASU, A., GAO, J., MITCHELL, J. \& SABHNANI, G. (2006) Distributed Localization Using Noisy Distance and Angle Information. ACM MobiHoc. Florence, Italy.

EREN, T., GOLDENBERG, D. K., WHITELEY, W., YANG, Y. R., MORSE, A. S., ANDERSON, B. D. O. \& BELHUMEUR, P. N. (2004) Rigidity, computation, and randomization in network localization. IEEE INFOCOM. Hong Kong, China.

FANG, J., CAO, M., MORSE, A. S. \& ANDERSON, B. (2009) Sequential Localization of Sensor Networks. SIAM Journal on Control and Optimization, 48, 321-350.

GOLDENBERG, D., BIHLER, P., CAO, M., FANG, J., ANDERSON, B., MORSE, A. S. \& YANG, Y. R. (2006) Localization in sparse networks using Sweeps. $A C M$ MobiCom. Los Angeles, CA.

GOLDENBERG, D. K., KRISHNAMURTHY, A., MANESS, W. C., YANG, Y. R. \& YOUNG, A. (2005) Network localization in partially localizable networks. IEEE INFOCOM. Miami, FL.

HORN, B. K. P., HILDEN, H. M. \& NEGAHDARIPOUR, S. (1988) Closed-form solution of absolute orientation using orthonormal matrices. Journal of the Optical Society of America A, 5, 1127-1135.

HOULE, M. E. \& TOUSSAINT, G. (1988) Computing the Width of a Set. IEEE Transactions on Pattern Analysis and Machine Intelligence, 5, 761-765.

KANNAN, A. A., FIDAN, B. \& MAO, G. (2008) Robust Distributed Sensor Network Localization Based on Analysis of Flip Ambiguities. IEEE GlobeCom.

KANNAN, A. A., FIDAN, B., MAO, G. \& D.O.ANDERSON, B. (2007) Analysis of Flip Ambiguities in Distributed Network Localization. IEEE Information, Decision and Control.

LEDERER, S., WANG, Y. \& GAO, J. (2008) Connectivity-based localization of large scale sensor networks with complex shape. IEEE INFOCOM. Phoenix, Arizona, USA.

LI, M. \& LIU, Y. (2009) Underground Coal Mine Monitoring with Wireless Sensor Networks. ACM Transactions on Sensor Networks (TOSN), 5.

LI, M. \& LIU, Y. (2010) Rendered Path: Range-Free Localization in Anisotropic Sensor Networks with Holes. IEEE/ACM Transactions on Networking (TON), 18 (1), 320-332.

LI, M., LIU, Y. \& CHEN, L. (2008) Non-Threshold based Event Detection for 3D Environment Monitoring in Sensor Networks. IEEE Transactions on Knowledge and Data Engineering (TKDE), 20, 1699-1711.

LIM, H. \& HOU, J. C. (2005) Localization for Anisotropic Sensor Networks. IEEE INFOCOM.

LIU, J., ZHANG, Y. \& ZHAO, F. (2006) Robust Distributed Node Localization with Error Management. ACM MobiHoc.
LIU, K., LI, M., LIU, Y., LI, M., GUO, Z. \& HONG, F. (2008) Passive Diagnosis for Wireless Sensor Networks. $A C M$ SenSys.

LIU, Y., CHEN, L., PEI, J., CHEN, Q. \& ZHAO, Y. (2007) Mining Frequent Trajectory Patterns for Activity Monitoring Using Radio Frequency Tag Arrays. IEEE PerCom.

LIU, Y., YANG, Z., WANG, X. \& JIAN, L. (2010) Location, Localization, and Localizability. Journal of Computer Science and Technology (JCST), 25(2), 274-297.

MO, L., HE, Y., LIU, Y., ZHAO, J., TANG, S. \& LI, X. (2009) Canopy closure estimates with GreenOrbs: Long-term large-scale sensing in the forest. ACM SenSys. Berkeley, California, USA.

MOORE, D., LEONARD, J., RUS, D. \& TELLER, S. J. (2004) Robust distributed network localization with noisy range measurements. ACM SenSys. Baltimore, MD.

NI, L. M., LIU, Y., LAU, Y. C. \& PATIL, A. (2004) LANDMARC: Indoor location sensing using active RFID. ACM Wireless Networks (WINET), 10, 701-710.

NICULESCU, D. \& NATH, B. (2004) Error characteristics of ad hoc positioning systems (APS). ACM MobiHoc.

PATWARI, N., ASH, J. N., KYPEROUNTAS, S., III, A. O. H., MOSES, R. L. \& CORREAL, N. S. (2005) Locating the Nodes: Cooperative localization in wireless sensor networks. IEEE Signal Processing Magazine, 54-69.

PRIYANTHA, N. B., BALAKRISHNAN, H., DEMAINE, E. D. \& TELLER, S. (2005) Mobile-Assisted Localization in Wireless Sensor Networks. IEEE Infocom.

SAVVIDES, A., GARBER, W., ADLAKHA, S., MOSES, R. \& SRIVASTAVA, M. B. (2003) On the error characteristics of multihop node localization in ad-hoc sensor networks. ACM/IEEE IPSN.

SAVVIDES, A., GARBER, W., MOSES, R. \& SRIVASTAVA, M. B. (2005) An analysis of error inducing parameters in multihop sensor node localization. IEEE Transactions on Mobile Computing, 4, 567-577.

SHANG, Y. \& RUML, W. (2004) Improved MDS-based localization. IEEE INFOCOM. Hong Kong, China.

WANG, X., LUO, J., LI, S., DONG, D. \& CHENG, W. (2008) Component Based Localization in Sparse Wireless Ad Hoc and Sensor Networks. IEEE ICNP.

WANG, Y., LEDERER, S. \& GAO, J. (2009) Connectivity-based Sensor Network Localization with Incremental Delaunay Refinement Method. IEEE INFOCOM.

WHITEHOUSE, K., WOO, A., KARLOF, C., JIANG, F. \& CULLER, D. (2005) The Effects of Ranging Noise on Multi-hop Localization: An Empirical Study. ACM/IEEE IPSN.

YANG, Z. \& LIU, Y. (2010) Quality of Trilateration: ConfidenceBased Iterative Localization. IEEE Transactions on Parallel and Distributed Systems (TPDS), 21, 631 - 640 